\title{
Defining the Collateral Flow of Posterior Tibial Artery and Dorsalis Pedis Artery in Ischemic Foot Disease: Is It a Preventing Factor for Ischemia?
}

\author{
Onur Tutar, ${ }^{1}$ Duzgun Yildirim, ${ }^{2}$ Cesur Samanci,, Babak Rafiee, ${ }^{1}$ Kaan Inan, ${ }^{3}$ Suleyman \\ Dikici, ${ }^{1}$ Fethi Emre Ustabasioglu, and Gokhan Kuyumcu ${ }^{1}$ \\ ${ }^{1}$ Department of Radiology, Cerrahpasa Faculty of Medicine, Istanbul University, Istanbul, Turkey \\ ${ }^{2}$ Centermed Advanced Imaging Center, Department of Radiology, Istanbul, Turkey \\ ${ }^{3}$ Department of Cardiovascular Surgery, Kasimpasa Military Hospital, Istanbul, Turkey \\ ${ }^{*}$ Corresponding author: Cesur Samanci, Department of Radiology, Cerrahpasa Faculty of Medicine, Istanbul University, Istanbul, Turkey. Tel: +90-5077511587, Fax: +90-2124143167, \\ E-mail:cesursamanci@gmail.com
}

Received 2014 July 7; Revised 2015 January 17; Accepted 2015 March 18.

\begin{abstract}
Background: Critical limb ischemia, a worldwide prevalent morbidity cause, is mostly secondary to vascular insufficiency due to atherosclerosis. The disease presents with intermittent claudication, which can progress to critical limb ischemia requiring amputation. Research has emphasized that the quality or existence of the pedal arch have a direct effect on wound healing and, therefore, on limb salvage, through the mechanism of collateral vascularization to the ischemic regions.

Objectives: This study aimed to determine the existence and, if present, grade of retrograde blood flow from plantar arch to dorsal foot artery (dorsalis pedis artery, DPA). The correlation between clinical symptoms and presence of collateral flow were also investigated.

Patients and Methods: Study group consisted of 34 cases, which included patient group ( $\mathrm{n}=17$, all male, mean age: 68 years) and control group ( $n=17$, all male, mean age: 66 years). After physical examination and lower extremity Doppler examination, spectral morphology of DPA flow was recorded, before and during manual compression of posterior tibial artery (PTA), for a period of 5 seconds. At the end, findings of Doppler ultrasound, computed tomography angiography, magnetic resonance angiography and, physical examination finding and symptomatology were gathered and analyzed.

Results: In the patient group, 31 lower limb arteries, of total of 17 cases, were included. After compression maneuver, DPA in 11 cases (six right, five left) showed retrograde filling from plantar arch. This retrograde flow support was triphasic in three cases, biphasic in five cases, and monophasic in three cases. In other DPAs of these 20 limbs, PTA based retrograde collateral flow was not determined. In nine of these 20 limbs, with no or diminished retrograde filling, symptoms were worse than in other cases. Contrarily, only two of 11 limbs, with retrograde collaterals, have claudication during walking.

Conclusion: In cases with critical atherosclerotic disease of anterior tibial artery, PTA-based biphasic or triphasic retrograde collateral flow prevents ischemia, whereas monophasic support or no retrograde flow remains incapable.
\end{abstract}

Keywords: Ultrasonography, Pulsed Doppler, Ischemia, Foot, Peripheral Arterial Disease, Atherosclerosis

\section{Background}

Critical limb ischemia, a worldwide prevalent morbidity cause, is mostly secondary to vascular insufficiency due to atherosclerosis (1). Symptomatology is mostly correlated with grade of atherosclerotic stenosis, in the infragenicular main crural arterial divisions. This disease mostly presents with intermittent claudication. In the final stages, it progresses to limb-threatening ischemia.

Even though medical treatments are promising, when critical limb ischemia symptoms progress, it is generally accepted that arterial revascularization is necessary. However, these surgical procedures become less efficient when stenosis is closer to the distal part of extremity and, generally, they are not readily applicable, because of the high surgical risk or contraindications $(2,3)$. On the other hand, newly emerging equipments and endovascular in- terventional alternatives are promising, as an alternative to surgical treatment of distal lesions (4-6).

As a very prevalent disease, lower limb atherosclerosis affects $3 \%$ of the entire population and, for the individuals over the age of 70, this figure rises to the level of $15 \%$ (7). Intermittent claudication may not be encountered in all of the cases. Moreover, this symptom does not always predict the presence or absence of peripheral arterial disease (PAD). Although it is stated that the effect of blood pressure, weight, and other risk factors may cause this ambiguity, an objective explanation has not been provided in the literature. In several studies, it is emphasized that the quality or existence of the pedal arch has a direct effect on wound healing and, therefore, on limb salvage $(7,8)$.

Copyright (C) 2016, Tehran University of Medical Sciences and Iranian Society of Radiology. This is an open-access article distributed under the terms of the Creative Commons Attribution-NonCommercial 4.0 International License (http://creativecommons.org/licenses/by-nc/4.0/) which permits copy and redistribute the material just in noncommercial usages, provided the original work is properly cited. 
Tutar $O$ et al.

\section{Objectives}

In this regard, we try to explain the degree of clinical findings and its relation with the degree of collateral development from plantar arch to dorsalis pedis artery (DPA) among cases having foot ischemia.

\section{Patients and Methods}

Permission was taken from the local institutional ethics committee, prior to the study. Verbal and written consent of each patient was taken in advance.

Totally, 34 male cases were included in this examination. A number of 17 of these cases comprised the patient group, with the mean age of 68 years. The subjects in the patient group comprised of patients referred to the Doppler ultrasonography (US) unit of our institution, due to exercise induced lower limb pain. In every case, measurements were recorded three times. Three cases, which did not show the same waveform and may cause intraobserver discrepancy, due to variation in collateral flow, or rare anatomical variation, were excluded from the study. Among the other 22 cases, who were considered as patient group after ultrasonography, only 17 cases, whose current vascular anatomy was evaluated and confirmed with computed tomography angiography (CTA) or magnetic resonance angiography (MRA), were enrolled in the study [CTA, $n=11$ and MRA, $n=6$; totally 31 lower extremities]. Consequently, other cases were excluded as they did not have confirmative CTA or MRA imagery. In these CTAs and MRAs, 17 of cases showed near critical degree of stenosis (50-70\%) in fibular artery (FA), patellar artery (PA), or anterior tibial artery (ATA) traces, which explains the symptoms and Doppler findings.

A number of 17 cases (34 lower extremities) were included, as healthy control group, with a mean age of 66 years. People were selected to the healthy control group randomly, among those whose both distal posterior tibial artery (PTA), ATA and DPA flows were homogeneous at spectral examination and had triphasic patterns.

After the selections, all cases underwent routine physical exam and lower extremity Doppler examination. The first intermetatarsal space was evaluated in a detailed manner during PTA compression test, to show retrograde collateral blood flow presence. Lower extremity Doppler US examinations of patient and control groups were done by the same observer (radiologist with 7 year-US Doppler experience), with the same device (Siemens ACUSON S2000, Siemens Healthcare, Erlangen, Germany), and under the same conditions (9 L Doppler transducer and $24 \mathrm{C}$ degree room temperature, supine position).

In this context, by localizing distal DPA and PTA and placing transducer parallel to the DPA course, through the first intermetatarsal space, a collateral color filling of ATA, sourced from the plantar arch, was searched. By using low pulse repetition frequency (PRF) values (3 - 5 $\mathrm{kHz}$ ), on triplex screen mode, with free left hand, and at the same time exact compression that cuts off the flow is applied to the distal PTA. The color and spectrum changes that occur on DPA trace were recorded at the level of ankle, for a period of 5 seconds.

For each examined extremity, in the patient group, all collected data and the elapsed time for claudication after walking were correlated. Relation between grade of collateral flow and amount of time, after which claudication begins, was statistically analyzed with Fisher exact test. The significance level considered in the study was $\mathrm{P}<0.05$.

\section{Results}

For the examined 31 lower extremities of 17 cases included, after compression maneuver, DPA in 11 cases (six right, five left), showed retrograde filling from plantar arch. This retrograde flow was triphasic in three cases, biphasic in five cases, and monophasic in three cases. In DPAs of other cases ( $n=20$ limbs), PTA based retrograde collateral flow was not determined. In this group, in nine cases, symptoms were worse than other cases. Contrarily, in 11 cases, in which collateral retrograde flow was present, only two limbs showed claudication and others showed a longer and painless walking distance.

In the 34 lower extremities of the control group, distal flow patterns were evaluated, as triphasic before compression maneuver and no post-stenotic flow pattern was defined. Doppler findings of left foot US of one case compared with right side and by confirmative CT angiography characteristics are shown (Figure 1). After PTA compression, just in one case, unilateral DPA flow showed monophasic conversion. Flow was not cut off exactly, in all other cases, and the distal triphasic pattern of the DPA continued, without any change. This case, only, had no lower limb symptoms during walking. Claudication was not observed in any of the cases in control group, too. As control group contained no cases with claudication, except for one case, which showed claudication like symptoms, without apparent cause, a correlation between symptomatology and collateral vessel presence was observed in patient group.

In the light of this information, to summarize more clearly the relationship between claudication and the presence of collaterals, in the group of patients with presence of collateral retrograde flow, two out of 11 lower limbs, developed pain, in less than 200 meters, although nine patients, out of 20 collateral free cases, developed pain.

Even though statistical findings were not significant $(P=0.135)$; this situation was probably related to the low number of cases. However, the presence of collateral pathways between PTA-DPA and its correlation with symptomology was apparent and $18 \%$ of all patient group had claudication, when walking 200 meters, however, $45 \%$ of cases, without such anastomosis, had claudication when walking the same distance. Results of statistical analysis were thought to be secondary to technical issues. Nevertheless, even if the statistical result was not significant, we think our data is very useful and convenient. 

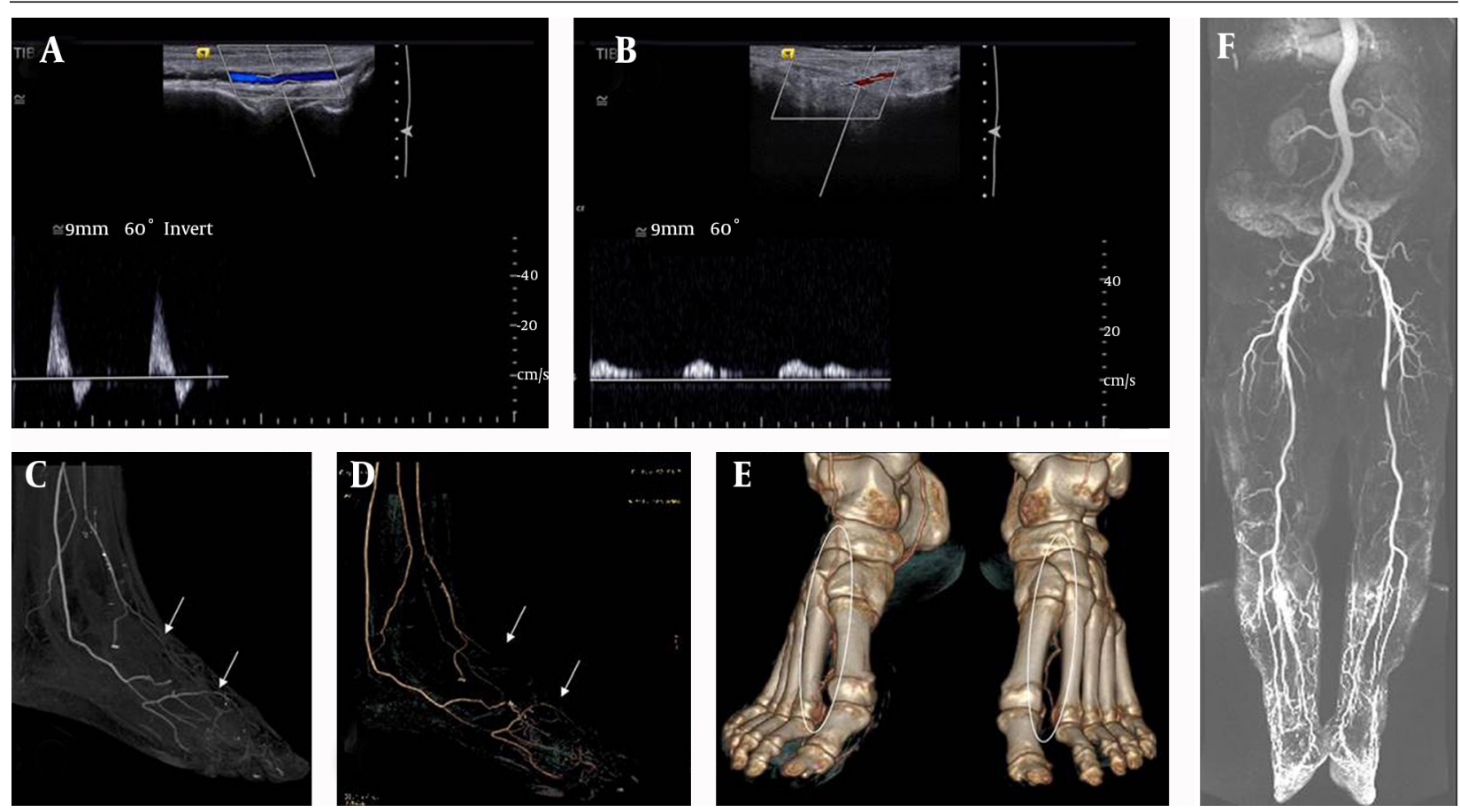

Figure 1. Doppler characteristics of left foot ultrasonography of one case compared with right side and by confirmative computed tomography angiography findings. A, Normal right dorsalis pedis artery biphasic spectral pattern; B, Abnormal, monophasic left dorsalis pedis artery pattern; C, Thick section maximum intensity projection (arrows); D, Three dimensional CT angiography (arrows); E, Volume rendered computed tomography images show the plantar arch support circulation at right side (ellipsoid area); F, High resolution contrast enhanced magnetic resonance angiography shows stenosis of the distal femoral artery, as the cause of left distal dampened-monophasic flow on Doppler window.

\section{Discussion}

The prevalence of arterial stenotic disease, in the lower extremity, reported in studies with larger samples ranged between $3-10 \%$ and over the age of 70 , severity of disease (causing claudication) could increase to $20 \%$ in the population $(2,3)$.

In this population, especially cases with diabetes, hyperlipidemia or smoking are examined by color Doppler US of the lower extremity arteries, for screening or for diagnosis of arterial disease. With Doppler US, evaluation of proximal arteries is much easier. Stenosis rates, intimalmedial complex anatomy and luminal flow patterns could be assessed more objectively. However, as the calibration of the vessels decreases toward the distal of the extremity, the spectral window and flow pattern, rather than lumen and wall anatomy, can be assessed more clearly on gray scale images (9).

In the literature, numerous studies are available about lower extremity arterial Doppler US examination and there are also many researches regarding spectrum information about examination techniques, calculation of stenosis degree, and many attempts to correlate different measurements types, like ankle-brachial index (ABI). Generally, sensitivity and specificity values of diagnosing the etiology of distal cruropedal ischemia have been evaluated at around $80 \%(10,11)$.

In our study, we discovered a new useful relationship, even in small arteries, which is difficult to assess with Doppler US. This new method can reveal the etiologic causes of symptoms and explain the severity of claudication, with high correlation. In addition, recent trends have focused excessively on the anatomic extent of the disease and arteriographic findings, without sufficient emphasis on the physiologic state of the limb (7).

Being the dominant PTA continuity, the lateral plantar artery may have branch(es) to the first intermetatarsal space, which have the potential to communicate with the distal DPA. Also, the embryological and anatomical relationship of this collateral pathway, presence or absence of distal ATA and distal PTA collateral circuit, is wellknown and could be a protective effect from ischemia. In their study, published in 2006, Attinger et al. stated that the blood flow to the foot and ankle is redundant, because the three major arteries feeding the foot have multiple intraarterial connnections (12). The "choke vessels' described by Taylor and Palmer illustrated a type of connection between different angiosomes; however, they also stated that several connections are true anastomoses, without a change in arterial calibre, using the example of the connection between the dorsalis pedis and the PTA to demonstrate this type of anatomosis (13).

In our cases, we have established the relationship between symptomatology and the spectral window pat- 
tern, after manual short-term occlusion of retrograde collateral flow, which comes from plantar arch into the distal DPA via first intermetatarsal space.

Our study has several limitations. The most important is a limited number of cases, because of not having a confirmative CTA or MRA for healthy group. Also, the expressions of patients were taken into account in claudication symptomatology, while no laboratory examination, body-mass index, ABI or smoking history were considered. First of all, because a number of cases will not have these demographical and clinical data, and they do not have an effect directly related with our examination results, we preferred not to turn this apprehensible study into a complicated one, with these unnecessary data. The limited number of cases results from the necessity of being examined by CTA or MRA, in patient group. On the other hand, the healthy group, to avoid exposing subjects to radiation or intravenous contrast, was not evaluated with cross-sectional examination. However, if necessary, examinations that were not requiring contrast administration, like arterial spin labelling, can be tested in further studies, with broad series. However, keeping in mind these limitations, as far as we know, there is no literature on the presence of PTA-DPA collateral connection. In this examination, the aim was to estimate presence or absence of collateral flow, and its relation with symptomatology, rather than the possibility of its relation with demographic findings.

Results of statistical analysis were thought to be secondary to technical issues. Nevertheless, even if the statistical result was not significant, we think our data is very useful and convenient.

In conclusion, we have seen retrograde collateral flow from plantar arch to distal DPA quite rarely in the healthy asymptomatic group, whereas it is more frequent in people who have atherosclerotic arterial disease. The existence of this collateral flow has a preventive effect on ischemia and reduces the presence of claudication. Therefore, in any Doppler examination of lower limb, this possible connection between PTA and DPA, via plantar arch, must be inquired in daily clinical routine.

\section{Footnotes}

Authors' Contribution:Study concept and design: Duzgun Yildirim, Onur Tutar, Cesur Samanci, Babak Rafiee, Kaan Inan, Suleyman Dikici, Fethi Emre Ustabasioglu, Acquisition of data: Duzgun Yildirim, Onur Tutar, Cesur Samanci, Kaan Inan, Suleyman Dikici, Fethi Emre Ustabasioglu, Analysis and interpretation of data: Duzgun Yildirim, Onur Tutar, Cesur Samanci, Babak Rafiee, Kaan Inan, Fethi Emre Ustabasioglu, Gokhan Kuyumcu, Drafting of the manuscript: Duzgun Yildirim, Onur Tutar, Cesur Samanci, Babak Rafiee, Fethi Emre Ustabasioglu, Gokhan Kuyumcu, Critical revision of the manuscript for important intellectual content: Duzgun Yildirim, Onur Tutar, Cesur Samanci, Babak Rafiee, Suleyman Di- kici, Fethi Emre Ustabasioglu, Statistical analysis: Cesur Samanci, Babak Rafiee, Fethi Emre Ustabasioglu, Gokhan Kuyumcu; Administrative, technical, and material support: Duzgun Yildirim, Onur Tutar,Cesur Samanci, Babak Rafiee, Suleyman Dikici, Fethi Emre Ustabasioglu, Study supervision: Duzgun Yildirim, Onur Tutar, Cesur Samanci, Kaan Inan, Fethi Emre Ustabasioglu, Gokhan Kuyumcu.

Financial Disclosure:The authors have no relevant financial interest in this article.

\section{References}

1. Faglia E, Clerici G, Clerissi J, Caminiti M, Quarantiello A, Curci V, et al. Angioplasty for diabetic patients with failing bypass graft or residual critical ischemia after bypass graft. Eur J Vasc Endovasc Surg. 2008;36(3):331-8. doi: 10.1016/j.ejvs.2008.04.012. [PubMed: 18538593]

2. Norgren L, Hiatt WR, Harris KA, Lammer J, Tasc Ii Working Group. TASC II section F on revascularization in PAD. J Endovasc Ther 2007;14(5):743-4. doi: 10.1583/1545-1550(2007)14[743:TISFOR]2.0. CO;2. [PubMed: 17924742]

3. Norgren L, Myhre HO. [Arterial insufficiency in the legs]. Tidsskr Nor Laegeforen. 2007;127(16):2123. [PubMed:17717581]

4. Adam DJ, Beard JD, Cleveland T, Bell J, Bradbury AW, Forbes $\mathrm{JF}$, et al. Bypass versus angioplasty in severe ischaemia of the leg (BASIL): multicentre, randomised controlled trial. Lancet. 2005;366(9501):1925-34. doi: 10.1016/s0140-6736(05)67704-5. [PubMed: 16325694]

5. Ferraresi R, Centola M, Ferlini M, Da Ros R, Caravaggi C, Assaloni R, et al. Long-term Outcomes after Angioplasty of Isolated, Below-the-knee Arteries in Diabetic Patients with Critical Limb Ischaemia. Eur J Vasc Endovasc Surg. 2009;37(3):336-42. doi: 10.1016/j.ejvs.2008.12.001. [PubMed:19112033]

6. Romiti M, Albers M, Brochado-Neto FC, Durazzo AES, Pereira $\mathrm{CAB}$, De Luccia N. Meta-analysis of infrapopliteal angioplasty for chronic critical limb ischemia. J Vasc Surg. 2008;47(5):975-981.e1. doi:10.1016/j.jvs.2008.01.005. [PubMed:18372148]

7. Mills JS, Conte MS, Armstrong DG, Pomposelli FB, Schanzer A Sidawy AN, et al. The Society for Vascular Surgery Lower Extremity Threatened Limb Classification System: risk stratification based on wound, ischemia, and foot infection (WIfI). J Vasc Surg. 2014;59(1):220-34 e1-2. doi: 10.1016/j.jvs.2013.08.003. [PubMed: 24126108]

8. Rashid H, Slim H, Zayed H, Huang DY, Wilkins CJ, Evans DR, et al. The impact of arterial pedal arch quality and angiosome revascularization on foot tissue loss healing and infrapopliteal bypass outcome. J Vasc Surg. 2013;57(5):1219-26. doi:10.1016/j. jvs.2012.10.129. [PubMed: 23523278]

9. Hiatt WR, Hirsch AT, Regensteiner JG, Brass EP. Clinical trials for claudication. Assessment of exercise performance, functional status, and clinical end points. Vascular Clinical Trialists. Circulation. 1995;92(3):614-21. [PubMed: 7634476]

10. Griffin KJ, Rankine J, Kessel D, Berridge DC, Scott DJA. Compression of the dorsalis pedis artery: a novel cause of blue toe syndrome. Vascular. 2012;20(6):325-8. doi: 10.1258/vasc.2011.cr0323. [PubMed: 22983548]

11. Roquer J, Segura T, Serena J, Castillo J. Endothelial dysfunction, vascular disease and stroke: the ARTICO study. Cerebrovasc Dis. 2009;27 Suppl 1:25-37. doi: 10.1159/000200439. [PubMed: 19342831]

12. Attinger CE, Evans KK, Bulan E, Blume P, Cooper P. Angiosomes of the foot and ankle and clinical implications for limb salvage: reconstruction, incisions, and revascularization. Plast Reconstr Surg. 2006;117(7 Suppl):261S-93S. doi: 10.1097/01. prs.0000222582.84385.54. [PubMed: 16799395]

13. Taylor GI, Palmer JH. The vascular territories (angiosomes) of the body: experimental study and clinical applications. Br J Plastic Surg. 1987;40(2):113-41. doi:10.1016/0007-1226(87)90185-8. 